\title{
Fluctuation Effects on Quadratic Autocatalysis Fronts
}

\author{
Mikhail V. Velikanov and Raymond Kapral \\ Chemical Physics Theory Group, Department of Chemistry, \\ University of Toronto, Toronto, Ontario M5S 3H6, Canada.
}

\begin{abstract}
A Markov chain model for spatially distributed autocatalytic systems with a quadratic reaction rate is considered. An approximate solution for the local probability distribution is obtained in the form of a perturbation expansion for the regimes where diffusion is relatively fast. Using this approximate distribution, properties of the chemical wave fronts found in these autocatalytic systems are studied, and deviations of the minimum propagation velocity and the concentration profile from deterministic predictions are analyzed. A comparison with numerical results from lattice-gas automaton simulations is also provided.
\end{abstract}

05.40.+j, 82.40.Bj, 82.20.Fd

\section{INTRODUCTION}

Chemical wave fronts are simple examples of spatiotemporal patterns supported by nonequilibrium chemical systems.t. The dynamics of such fronts is generally described by a set of deterministic reaction-diffusion equations; a wave front is defined as a special solution of these equations, unformly translating in space with constant velocity and connecting two distinct homogeneous stationary states. In particular, the wave front solutions of the reaction-diffusion equation,

$$
\frac{\partial c}{\partial t}=\nabla^{2} c+c(1-c)
$$

have been studied extensively. Here $c$ is the (scalar) order parameter field ( $c$ varies between 0 and 1 ), and $t$ is the time variable. Equation 1 , was first considered by Fisher and Kolmogorov et al. in the context of population dynamics and has since found applications in a variety of other disciplines, including nonlinear chemical kinetics. 10 From a chemical viewpoint, equations of this type describe the evolution of a spatially distributed autocatalytic system with a quadratic reaction rate (referred to in this paper as a quadratic autocatalysis system).

It is well known that, given appropriate initial conditions, eq. (1) admits (one-dimensional) wave front solutions of the form $c=c(x-v t)$, where $v$ is the front velocity and $x$ is the spatial coordinate in the direction of front movement. The front velocity must not be less than the minimum yelocity $v_{\min }=2$ in order for the wave front to exist. 19 Further analysis (see ref.11 for details) shows that the solution with $v=v_{\min }$ is marginally stable and, therefore, any wave front initiated with a sufficiently steep initial profile will eventually relax to this minimum-velocity solution. No exact analytic solution of eq. (11) is known for general $v$; however, it is possible to derive the following approximate form for the front profile, which is uniformly valid for $v \geq v_{\min }$ :

$$
c(z)=\frac{1}{1+e^{z / v}}+\frac{e^{z / v}}{v^{2}\left(1+e^{z / v}\right)^{2}} \ln \left[\frac{4 e^{z / v}}{\left(1+e^{z / v}\right)^{2}}\right],
$$

where $z=x-v t$ is the spatial coordinate of the reference frame moving with the wave front.

This deterministic description, although capable of capturing the gross features of the front dynamics, is less successful with respect to finer details. For instance, reaction-diffusion equations do not provide an adequate framework for understanding the influence of fluctuations intrinsic to the local reactive and diffusive dynamics on the macroscopic properties of the wave front. This is due to the fact that reaction-diffusion equations are essentially mean-field equations in which all correlations built by the local fluctuating dynamics are neglected. However, recent numerical studies on chemical fronts have shown that fluctuations significantly affect both the propagation velocity and the concentration profile of the wave front 12.17

In this paper we present a theory which systematically accounts for the effects of fluctuations on the properties of the wave fronts in quadratic autocatalysis systems. Our analysis is based on the perturbation theoretic formalism developed earlier and applied to an oscillatory reaction-diffusion system 18 The system is described by a Markov chain model19. 20 whose mean-field kinetic equation is the generalized Fisher-Kolmogorov equation We use a Chapman-Enskog-like perturbative technique21 to extract the corrections to the mean-field local probability distribution due to fluctuations in the local dynamics. The formal expansion parameter $\gamma$ gauges the ratio of characteristic time scales of diffusion and reaction processes, and is small when diffusion is fast enough, i.e. the 
deviation from the mean-field behaviour is small. The kinetic equation for the local concentration obtained from the generalized local distribution contains a reaction rate term which is not quadratic in the concentration. We further analyze the wave front solutions of the generalized kinetic equation and determine the dependence of the front properties on the diffusion coefficient. We also compare these results with the deterministic predictions and the data obtained in lattice-gas automaton simulations of the quadratic autocatalysis system.

The paper is organized in the following manner. In Sec. II we introduce the Markov chain model for quadratic autocatalysis systems and review the meanfield results for the properties of the wave fronts in this model. In Sec. II, using the perturbative technique developed in Ref. $\$$ the wave fronts are analyzed in a framework that goes beyond mean-field theory. The dependence of the minimum propagation velocity and the front shape on the diffusion coefficient, including the effects of fluctuations, is derived and compared to the mean-field results. Section IV presents the results of lattice-gas automaton simulations of a quadratic autocatalysis system and compares these results with the predictions of the perturbation theory. Finally, Sec. V contains a discussion of the results of our study.

\section{MARKOV CHAIN MODEL}

We consider a system which consists of a chemical species, $A$, diffusing and reacting in solution. We assume a discrete-space, discrete-time description of the system in which space is partitioned into cells of fixed volume and time into intervals of unit length. The cells can be alternatively viewed as nodes of a lattice; reactant particles residing at a node may react according to the reaction mechanism, with probability determined by combinatorial rules, or execute diffusive jumps to the neighboring nodes. Furthermore, we impose exclusion principle, according to which a node cannot be occupied by more than $N$ reactant particles.

The following "particle-hole" reaction mechanism is used in this model:

$$
\begin{array}{r}
A+A^{*} \stackrel{k_{1}}{\rightarrow} 2 A, \\
A \stackrel{k_{2}}{\rightarrow} \emptyset .
\end{array}
$$

Here $k_{1}$ and $k_{2}$ are rate coefficients and an asterisk denotes a vacancy, i.e. an empty space at a node that can be occupied by a particle of $A$. The concentrations of pool chemical species (i.e. species whose concentrations are fixed by constraints) are incorporated in the rate constants. Reactions of this type that depend on the concentrations of both particles and particle vacancies are common in surface chemistry and in biochemistry. The solvent particles are assumed to be chemically inert. At any time the state of the system is completely specified by the number of the particles of $A$ occupying every node.
In the following, we set $k_{1}=1$; one can easily show that this is always possible by appropriate rescaling of time. The probabilities per unit time of the two reactions, determined by the combinatorics, are, respectively,

$$
p_{1}=\frac{h}{N-1} a(\mathbf{r})(N-a(\mathbf{r})), p_{2}=h k_{2} a(\mathbf{r})
$$

Here $h$ is a parameter that sets the time scale of the Markov chain, and $a(\mathbf{r})$ is the total number of particles at the node with position vector $\mathbf{r}$. The factor $(N-1)^{-1}$ is included so that the mean-field kinetic equation (i.e. reaction-diffusion equation) for the concentration of $A$ can be written in a neat analytic form (see discussion below).

The inert solvent particles serve to randomize of the diffusive jumps that the reactant particles execute. The probability that a particle at the node with position vector $\mathbf{r}$ will execute a diffusive jump within a unit time interval is equal to the ratio $a(\mathbf{r}) / N$. The direction of the jumps is selected at random for each time interval and is the same for all nodes in the system. One can show that this diffusion mechanism yields the correct form of the diffusion equation for the local concentration.

The Markov chain which describes evolution of the full probability distribution function is constructed from the successive application of the reaction and diffusion Markov chains. The numerical simulations reported in Sec. IV utilize this full Markov chain, without any further assumption or simplification.

\section{A. Local Markov chain dynamics}

To facilitate theoretical analysis of the Markov chain model an additional assumption is emplpyed. Numerical studies of the diffusion Markov chain18.22 showed that for systems with spatial dimensions two or greater the diffusion rule described above leads to rapid de-correlation of the particle number densities at different spatial locations. Consequently, the full probability distribution for the entire system may be factored into a product of single-node probability distributions. In the following, we assume that the system's dimensionality is large enough so that this factorization approximation is valid and, for sufficiently long times, the de-correlation allows description of the evolution in terms of the local probability distribution function $P(a(\mathbf{r}), n)$.

The evolution is due to two competing processes, namely local reactive events and diffusive transport of particles. Correspondingly, the evolution equation for the local distribution function $P(a(\mathbf{r}), n)$ can be written in the following form: 


$$
P(a(\mathbf{r}), n+1)-P(a(\mathbf{r}), n)=\left(\gamma \hat{W}^{R}+\hat{W}^{D}\right) P(a(\mathbf{r}), n) .
$$

Here $n$ is the (integer) time variable and $\hat{W}^{D}$ and $\hat{W}^{R}$ are the evolution operators for pure diffusion and reaction processes, respectively. The dimensionless parameter $\gamma$ gauges the relative contributions of these two processes to the overall dynamics. A natural choice for the defini- tion of $\gamma$ is the ratio of characteristic time scales of the diffusion and reaction processes.

The matrix elements of the diffusion evolution operator $\hat{W}^{D}$ are 22

$$
\begin{aligned}
W_{a(\mathbf{r}), a^{\prime}(\mathbf{r})}^{D} & =\frac{\chi(\mathbf{r}, n)}{N}\left(1-\frac{a^{\prime}(\mathbf{r})}{N}\right) \delta_{a^{\prime}(\mathbf{r}), a(\mathbf{r})-1}+\frac{a^{\prime}(\mathbf{r})}{N}\left(1-\frac{\chi(\mathbf{r}, n)}{N}\right) \delta_{a^{\prime}(\mathbf{r}), a(\mathbf{r})+1} \\
& -\left[\frac{\chi(\mathbf{r}, n)}{N}\left(1-\frac{a^{\prime}(\mathbf{r})}{N}\right)+\frac{a^{\prime}(\mathbf{r})}{N}\left(1-\frac{\chi(\mathbf{r}, n)}{N}\right)\right] \delta_{a^{\prime}(\mathbf{r}), a(\mathbf{r})} .
\end{aligned}
$$

Here $\chi(\mathbf{r}, n)$ is the mean particle number density (i.e. concentration) averaged over the immediate neighbourhood of node $\mathbf{r}$, i.e.

$$
\chi(\mathbf{r}, n)=\frac{1}{m} \sum_{\mathbf{r}^{\prime} \in \mathcal{N}(\mathbf{r})} \bar{a}\left(\mathbf{r}^{\prime}, n\right),
$$

where $m$ is the coordination number of the lattice, $\mathcal{N}(\mathbf{r})$ is the neighbourhood of node $\mathbf{r}$ and $\bar{a}\left(\mathbf{r}^{\prime}, n\right)$ is concentration at node $\mathbf{r}^{\prime}$ at time $n$,

$$
\bar{a}\left(\mathbf{r}^{\prime}, n\right)=\sum_{a\left(\mathbf{r}^{\prime}\right)} a\left(\mathbf{r}^{\prime}\right) P\left(a\left(\mathbf{r}^{\prime}\right), n\right) .
$$

One can show that the stationary local distribution for the $\hat{W}^{D}$ operator alone is binomial,

$$
P_{s}^{D}(a(\mathbf{r}))=\left(\begin{array}{c}
N \\
a(\mathbf{r})
\end{array}\right)\left(\frac{\bar{a}(\mathbf{r})}{N}\right)^{a(\mathbf{r})}\left(1-\frac{\bar{a}(\mathbf{r})}{N}\right)^{N-a(\mathbf{r})}
$$

The matrix elements of the reaction evolution opera- $\quad$ tor $\hat{W}^{R}$ are

$$
\begin{aligned}
W_{a(\mathbf{r}), a^{\prime}(\mathbf{r})}^{R} & =\frac{h}{N-1} a^{\prime}(\mathbf{r})\left(N-a^{\prime}(\mathbf{r})\right) \delta_{a^{\prime}(\mathbf{r}), a(\mathbf{r})-1}+h k_{2} a^{\prime}(\mathbf{r}) \delta_{a^{\prime}(\mathbf{r}), a(\mathbf{r})+1} \\
& -\left[\frac{h}{N-1} a^{\prime}(\mathbf{r})\left(N-a^{\prime}(\mathbf{r})\right)+h k_{2} a^{\prime}(\mathbf{r})\right] \delta_{a^{\prime}(\mathbf{r}), a(\mathbf{r})} .
\end{aligned}
$$

Note that the operator $\hat{W}^{R}$ satisfies the exclusion principle by construction since the probability of creating a particle of $A$ at a fully occupied node vanishes.

If $k_{2}>0$, the stationary distribution for the pure reaction Markov chain is $\delta_{a(\mathbf{r}), 0}$. This strongly correlated distribution is the consequence of the absorbing boundary at $a(\mathbf{r})=0$ inherent in the pure reaction dynamics. Indeed, as one can see from (7), once a system evolving under the pure reaction Markov chain reaches the state with $a(\mathbf{r})=0$ (i.e. totally empty), it remains trapped in that state for all future times. In the special case $k_{2}=0$, there is an additional absorbing boundary at
$a(\mathbf{r})=N$, hence the stationary distribution has the form $n \delta_{a(\mathbf{r}), 0}+l \delta_{a(\mathbf{r}), N}$, where $n$ and $l$ are constants satisfying $n+l=1$ and determined by the initial conditions.

In a spatially-distributed system with finite diffusion coefficient, the analytic form of the local probability distribution is determined by the interplay between the reactive and diffusive processes described by the operators $\hat{W}^{R}$ and $\hat{W}^{D}$. Namely, as the diffusion coefficient increases, the local distribution tends to the uncorrelated binomial form prescribed by the pure diffusion process, with time dependence incorporated trivially through the mean particle density, viz 


$$
P_{B}(a(\mathbf{r}), n)=\left(\begin{array}{c}
N \\
a(\mathbf{r})
\end{array}\right)\left(\frac{\bar{a}(\mathbf{r}, n)}{N}\right)^{a(\mathbf{r})}\left(1-\frac{\bar{a}(\mathbf{r}, n)}{N}\right)^{N-a(\mathbf{r})}
$$

Conversely, as the diffusion coefficient tends to zero, correlations characteristic of the stationary distribution of the pure reaction Markov chain contaminate the binomial form (8) to an increasing degree. The subject of this paper is the effect of these correlations on the properties of the macroscopic structures, such as wave fronts, existing in the system.

\section{B. Mean-field dynamics}

Consider the mean-field dynamics of the reactiondiffusion Markov chain. The mean-field local distribution function is the time-dependent binomial (8); the kinetic equation for concentration of $A$ can be easily obtained using it and eq. (3); after some analysis, we find

$$
\begin{aligned}
\bar{a}(\mathbf{r}, n+1)-\bar{a}(\mathbf{r}, n) & =\sum_{a=0}^{N} a(\mathbf{r})\left(\hat{W}^{D}+\gamma \hat{W}^{R}\right) P_{B}(a(\mathbf{r}), n) \\
& =\frac{1}{m N} \Delta \bar{a}(\mathbf{r}, n)+\gamma h \bar{a}(\mathbf{r}, n)\left(\beta-\frac{\bar{a}(\mathbf{r}, n)}{N}\right) \\
& \equiv \tilde{D} \Delta \bar{a}(\mathbf{r}, n)+\gamma R(\bar{a}(\mathbf{r}, n)) .
\end{aligned}
$$

Here $\beta=1-k_{2}, \tilde{D}=(m N)^{-1}, \Delta$ is the discrete Laplacian operator and $R(\bar{a}(\mathbf{r}, n))$ denotes the mass-action law terms. Introducing the rescaled variables,

$$
h \rightarrow \gamma \beta h, \quad \bar{a}(\mathbf{r}, n) \rightarrow \frac{\bar{a}(\mathbf{r}, n)}{N \beta},
$$

and the length unit, $L=\sqrt{h / \tilde{D}}$, one can rewrite eq. (9) as follows:

$$
\bar{a}(\mathbf{r}, n+1)-\bar{a}(\mathbf{r}, n)=\frac{h}{L^{2}} \Delta \bar{a}(\mathbf{r}, n)+h \bar{a}(\mathbf{r}, n)(1-\bar{a}(\mathbf{r}, n)) .
$$

Clearly, eq. (10) is just a discretized form of eq. (11).

\section{PERTURBATION THEORY FOR THE WAVE FRONT SOLUTIONS}

We now turn our attention to the analysis of the evolution equation (3). We derive an approximate kinetic equation for the local concentration field valid for small $\gamma$ and analyze the properties of its wave front solutions.

\section{A. Local concentration dynamics for $\gamma \ll 1$}

We seek the solution of eq. (3) in the form of a regular perturbation series with time-dependent binomial distribution as the leading term,

$$
P(a(\mathbf{r}), n)=P_{B}(a(\mathbf{r}), n)+\sum_{k=1}^{\infty} \gamma^{k} P_{k}(a(\mathbf{r}), n) .
$$

In the following, we will keep only the leading and firstorder terms of the series (11). The starting point of our analysis is the evolution equation for the first-order term, $P_{1}(a(\mathbf{r}), n)$, which is obtained by substituting eq. (11) into eq. (3), collecting terms of similar order in $\gamma$ and using the solvability conditions (cf. eq. (20) of Ref.18 and discussion therein), viz

$$
P_{1}(a(\mathbf{r}), n+1)-P_{1}(a(\mathbf{r}), n)=\hat{W}^{D} P_{1}(a(\mathbf{r}), n)+\hat{S} P_{B}(a(\mathbf{r}), n),
$$


where the operator $\hat{S}$ is defined as follows:

$$
\hat{S}=\hat{W}^{R}-R(\bar{a}(\mathbf{r}, n)) \frac{\partial}{\partial \bar{a}}
$$

Suppose that $P_{1}(a(\mathbf{r}), n)$ changes slowly with time. Then, we can write the formal solution of eq. (12) as

$$
P_{1}(a(\mathbf{r}), n)=-\left(\hat{W}^{D}\right)^{-1} \hat{S} P_{B}(a(\mathbf{r}), n),
$$

where $\left(\hat{W}^{D}\right)^{-1}$ is the operator reciprocal to $\hat{W}^{D}$. Using (14) and (3), one can now determine the dynamics of the local concentration and we obtain

$$
\begin{aligned}
\bar{a}(\mathbf{r}, n+1)-\bar{a}(\mathbf{r}, n) & =\sum_{a(\mathbf{r})=0}^{N} a(\mathbf{r})\left(\gamma \hat{W}^{R}+\hat{W}^{D}\right)\left(P_{B}(a(\mathbf{r}), n)+\gamma P_{1}(a(\mathbf{r}), n)\right) \\
& =\frac{1}{m N} \Delta \bar{a}(\mathbf{r}, n)+\gamma R(\bar{a}(\mathbf{r}))-\gamma^{2} \sum_{a(\mathbf{r})=0}^{N} a(\mathbf{r}) \hat{W}^{R}\left(\hat{W}^{D}\right)^{-1} \hat{S} P_{B}(a(\mathbf{r}), n) .
\end{aligned}
$$

The calculation of the $\mathcal{O}\left(\gamma^{2}\right)$ correction term in eq.

$$
\sum_{a(\mathbf{r})=0}^{N} a(\mathbf{r}) \hat{W}^{R}\left(\hat{W}^{D}\right)^{-1} \hat{S} P_{B}(a(\mathbf{r}), n)=\frac{N h^{2}}{N-1} \bar{a}(\mathbf{r}, n)\left(1-\frac{\bar{a}(\mathbf{r}, n)}{N}\right)^{2} .
$$

The final form of the kinetic equation for $\bar{a}(\mathbf{r}, n)$ is obtained by substituting (16) into (15) and transforming the concentration variable, $\bar{a}(\mathbf{r}, n) \rightarrow \bar{a}(\mathbf{r}, n) / N$. More- over, at this point we can set the ordering parameter $\gamma$ to unity. In this way we come to

$$
\bar{a}(\mathbf{r}, n+1)-\bar{a}(\mathbf{r}, n)=\tilde{D} \Delta \bar{a}(\mathbf{r}, n)+h \bar{a}(\mathbf{r}, n)(\beta-\bar{a}(\mathbf{r}, n))-\frac{N h^{2}}{N-1} \bar{a}(\mathbf{r}, n)(1-\bar{a}(\mathbf{r}, n))^{2}
$$

where $\beta=1-k_{2}$. Note that eq. (17) can be derived from the exact solution of (12) by an asymptotic expansion for small $\gamma$ of the integral terms in the corresponding kinetic equation (cf. eq. (25) of Ref.18 and discussion that fol- lows).

For any fixed $h$, eq. (17) can be viewed as a discretized version of the following partial differential equation (written here for the case of a planar front):

$$
\frac{\partial \bar{a}(x, t)}{\partial t}=D \frac{\partial^{2} \bar{a}(x, t)}{\partial x^{2}}+\bar{a}(x, t)(\beta-\bar{a}(x, t))-K \bar{a}(x, t)(1-\bar{a}(x, t))^{2},
$$

where $D=(m N h)^{-1}, K=\frac{N h}{N-1}$. Note that, as defined, $K$ is inversely proportional to the diffusion coefficient $D$, $K=\alpha / D$, where $\alpha=\frac{1}{m(N-1)}$.
Equation (18) can be written in terms of the same variables as the mean-field reaction-diffusion equation (10), with a rescaling of space, $x \rightarrow x / \sqrt{D}$, as follows:

$$
\frac{\partial \bar{a}(x, t)}{\partial t}=\frac{\partial^{2} \bar{a}(x, t)}{\partial x^{2}}+\bar{a}(x, t)(\beta-\bar{a}(x, t))-\frac{\alpha}{D} \bar{a}(x, t)(1-\bar{a}(x, t))^{2}
$$


The reaction rate in eq. $(\sqrt{19})$ is not quadratic in $\bar{a}(x, t)$, in contrast to eq. (10), because of the $\mathcal{O}\left(D^{-1}\right)$ correction term. For convenience, all further analysis will be carried out using the continuous-space, continuous-time equation (19) rather than the finite-difference equation (17). Since eq. (17) is obtained from eq. (19) by Euler discretization and a linear transformation of variables, both equations describe essentially the same dynamics.

\section{B. Analysis of the wave front solutions}

Following the standard formalism, 1, 8, 8 we recast eq. (19) in terms of the variable $z$, the coordinate of a reference frame moving with the wave front, i.e. $z=x-v t$,

$$
\frac{d^{2} \bar{a}}{d z^{2}}+v \frac{d \bar{a}}{d z}=\bar{a}(\bar{a}-\beta)+\frac{\alpha}{D} \bar{a}(1-\bar{a})^{2} .
$$

Here we will consider only wave fronts moving to the right $(v>0)$; generalization of our results to the left-moving fronts is trivial. Letting $p=d \bar{a} / d z$, we can rewrite eq. (20) as a system of first-order ODEs,

$$
\left\{\begin{array}{l}
\dot{\bar{a}}=p, \\
\dot{p}=-v p+\bar{a}(\bar{a}-\beta)+\frac{\alpha}{D} \bar{a}(1-\bar{a})^{2},
\end{array}\right.
$$

where the dot indicates differentiation with respect to $z$. This system posesses up to three fixed points, one at the origin of the phase plane and the other two at $(\bar{a}, p)=\left(\bar{a}_{1 s}, 0\right)$ and $\left(\bar{a}_{2 s}, 0\right)$, where

$$
\begin{aligned}
& \bar{a}_{1 s}=1-\frac{D-\sqrt{D^{2}+4 \alpha(\beta-1) D}}{2 \alpha}, \\
& \bar{a}_{2 s}=1-\frac{D+\sqrt{D^{2}+4 \alpha(\beta-1) D}}{2 \alpha} .
\end{aligned}
$$

From a linear stability analysis, we find the following relation for the eigenvalues associated with the fixed points:

$$
\mu^{2}+v \mu-\frac{\alpha}{D}(1-\bar{a})(1-3 \bar{a})-2 \bar{a}+\beta=0,
$$

where $\bar{a}=0, \bar{a}_{1 s}$, or $\bar{a}_{2 s}$. The diagram in Fig. 1 summarizes the behaviour of the fixed-point structure with respect to $D$ and $\beta$, as determined by eqns. (22) and (23). The four zones in the diagram correspond to different linear stability properties of the fixed points as follows:

zone I: only the fixed point at the origin exists and is unstable, zone II: the point at the origin is unstable, the point at $\bar{a}_{1 s}$ is unstable and moves away from the origin as $D$ increases $\left(\bar{a}_{1 s}>0\right)$, the point at $\bar{a}_{2 s}$ is stable and moves toward the origin as $D$ increases $\left(\bar{a}_{2 s}>0\right)$,

zone III: the point at the origin is unstable, the point at $\bar{a}_{1 s}$ is stable and moves toward the origin as $D$ increases $\left(\bar{a}_{1 s}<0\right)$, the point at $\bar{a}_{2 s}$ is unstable and moves away from the origin as $D$ increases $\left(\bar{a}_{2 s}<0\right)$, and

zone IV: the point at the origin is stable, the point at $\bar{a}_{1 s}$ is unstable and moves away from the origin as $D$ increases $\left(\bar{a}_{1 s}>0\right)$, the point at $\bar{a}_{2 s}$ is unstable and moves away from the origin as $D$ increases $\left(\bar{a}_{2 s}<0\right)$.

The two lines that define these zones are: a) $D=$ $4 \alpha(1-\beta)$, the line of parameter values at which the fixed points at $\bar{a}_{1 s}$ and $\bar{a}_{2 s}$ first emerge as a stable, degenerate, zero-eigenvalue node (dashed line), and b) $D=\alpha / \beta$, the line where the fixed point at the origin changes its stability (solid line). We observe that formation of a wave front replacing the steady state at the origin with the steady state at $\bar{a}_{1 s}$ is possible if the diffusion coefficient is greater than the critical value $D_{\mathrm{cr}}=\alpha / \beta$. Indeed, in that region of the diagram the fixed point at the origin is stable, the point at $\bar{a}_{1 s}$ is unstable (with $\bar{a}_{1 s}>0$ ), and $\bar{a}_{2 s}$ is negative. Moreover, it can be easily seen that $\bar{a}_{1 s}$ tends to $\beta$ as $D$ increases. Thus, in the limit $D \rightarrow \infty$ the fixed-point structure of (21) is equivalent to that of eq. (10) of Sec. II, save for additional fixed point in an unphysical area of the phase plane. 


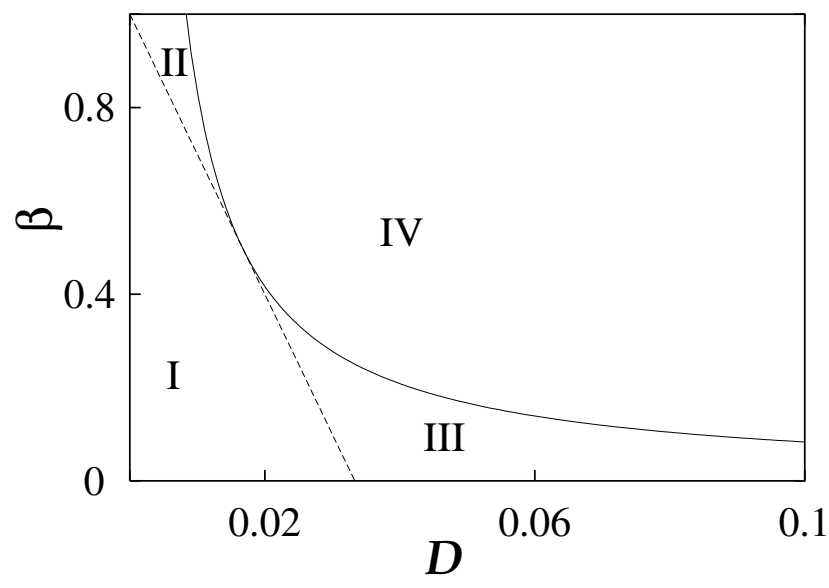

FIG. 1. Diagram of the fixed-point structure of the ODE system (21) as a function of $D$ and $\beta$, with $\alpha=\frac{1}{120}$. The critical diffusion coefficient line $D_{\mathrm{cr}}=\alpha / \beta$ is indicated by a solid line. The dashed line denotes the locus of $(D, \beta)$ values where the movable fixed points first emerge as a degenerate node. For description of the fixed-point behaviour in the different zones in the diagram, see text above.

In order to represent a physically meaningful state of the system, a wave front solution of (21) with $0<\beta<1$ must exhibit no oscillatory behaviour near the leading edge of the front. This implies that both roots of eq. (23) with $\bar{a}=0$ should be real. One can show by elementary analysis that this requirement is satisfied if

$$
v \geq 2 \sqrt{\beta-\frac{\alpha}{D}} .
$$

For any finite $D$ the minimum propagation velocity given by (24) and the concentration at the upper plateau of the wave front $\left(\bar{a}_{1 s}\right.$ in eq. $\left.(22)\right)$ are both less than their respective values predicted by the mean-field theory and converge to those values as $D \rightarrow \infty$.

\section{NUMERICAL RESULTS}

In order to test the accuracy of the results obtained in the preceding section, we numerically solved the full Markov chain dynamics using a lattice-gas automaton algorithm. The simulations were performed on a thin $(4 \times 3000$ nodes $)$ strip of a 2 -dimensional triangular lattice; wave fronts were generated from a step-like initial concentration profile placed in the middle of the lattice. The value of the rate coefficient $k_{2}$ used in these simulations was 0.333 , so that $\beta=0.667$. The exclusion parameter was fixed at $N=21$ which, together with the coordination number for triangular lattice $(m=6)$, gives $\alpha=\frac{1}{120}$. The value of the diffusion coefficient $D=(m N h)^{-1}$ varied between 0.1 and 1.0. All of the front properties were measured using the mean concentration profile obtained by averaging over 10 realizations of the dynamics. Sufficient time was allowed to elapse before the measurements were taken to ensure that the wave front relaxed to its stable, uniformly propagating form. In our simulations, the period of transient dynamics was found to be relatively long $\left(\sim 10^{5}\right.$ automaton time steps). Within this period, the front width was observed to grow diffusively; however, no significant growth in the front width was detected after the transient regime.

In Fig. 2 we compare the front velocity observed in these simulations as a function of the diffusion coefficient $D$ with the minimum propagation velocity predicted on the basis of the present theory (eq. (24)) and the meanfield, reaction-diffusion equation (10).

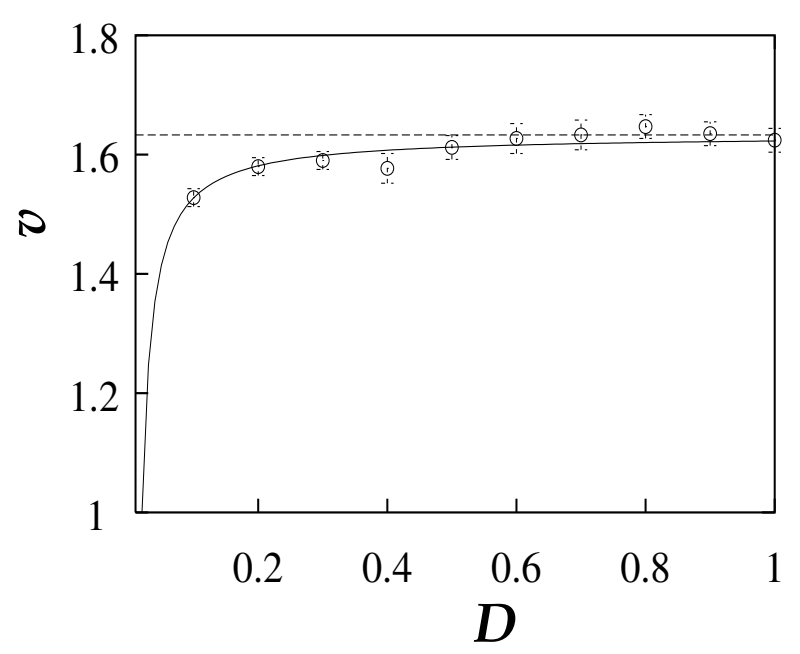

FIG. 2. Dimensionless front velocity $v$ versus the diffusion coefficient $D$. Circles denote the data obtained in lattice-gas automaton simulations. The minimum propagation velocity given by the present theory (eq. (24)) is plotted with a solid line. The results of the mean-field theory are indicated by a dashed line. Parameters $\alpha$ and $\beta$ are equal to $\frac{1}{120}$ and 0.667 , respectively.

We observe that the propagation velocity predicted by the present theory is in good agreement with the data from the numerical simulations.

\section{CONCLUSIONS}

The generalized kinetic equation derived in this study admits travelling wave solutions whose properties are different from the predictions of mean-field theory. There is a non-zero critical value of the diffusion coefficient below which no stable wave front solution exists. Moreover, the concentration at the upper plateau of the wave front varies with the diffusion coefficient as well as the kinetic parameters of the system. Most significantly, the (dimensionless) minimum front velocity decreases as the diffusion coefficient is decreased. These effects have their origin in the local correlations built by the fluctuating reactive dynamics which persist for finite values of the diffusion coefficient. 
For finite values of the diffusion coefficient, measurements of the front velocity in two-dimesional lattice-gas simulations of the full Markov chain model confirmed that the present theory describes the departure from the mean-field results with quantitative accuracy. Similar simulations were also carried out on one-dimensional systems. The results of these simulations showed the same qualitative trend as in two dimensions. However, because the primary assumption of our theory (the factorization of the full probability distribution) is not valid for one spatial dimension, the quantitative agreement between theoretical data and the simulation results is much poorer than that for two dimensions.

There is a number of numerical studie $12,42.23$ on the breakdown of mean-field descriptions for quadratic autocatalysis fronts using a variety of microscopic models, none of which is the same as that employed in our study. The lattice-gas automaton simulations of Lemarchand et al. 12 have shown that the mean-field results remain valid for the range of diffusion coefficient values used in their study. Later investigations using a Langevin equation approach reported an increase in the front velocity above the mean-field value.13.14 Monte Carlo studies of Riordan et al. have focused on the front width and have found that the width grows algebraically in one and two space dimensions, while in higher dimensions the width dynamics is adequately described by mean-field theory.23 In our simulations on two-dimensional systems with small transverse size, the front width grows diffusively during a very long transient period but then saturates and the front evolves without change in width. The effects of microscopic fluctuations on quadratic autocatalyis fronts clearly present a number of interesting features.

\section{ACKNOWLEDGMENTS}

This work was partially supported by the Natural Sciences and Engineering Research Council of Canada. M.V. also benefits from a Connaught Scholarship. Computing resources for this study were provided in part by the University of Toronto Information Commons.

\section{APPENDIX}

To compute the $\mathcal{O}\left(\gamma^{2}\right)$ correction term in eq. 15, note the following properties of operator $\hat{W}^{D}$ (the proof can be obtained by straightforward calculation, as in Appendix A of Ref18):

$$
\begin{aligned}
\sum_{a(\mathbf{r})=0}^{N} a(\mathbf{r})\left(\hat{W}^{D}\right)^{p} X(a(\mathbf{r}), n) & =\left(-\frac{1}{N}\right)^{p} \sum_{a(\mathbf{r})=0}^{N} a(\mathbf{r}) X(a(\mathbf{r}), n), \\
\sum_{a(\mathbf{r})=0}^{N} a^{2}(\mathbf{r})\left(\hat{W}^{D}\right)^{p} X(a(\mathbf{r}), n) & =\left(-\frac{2}{N}\right)^{p} \sum_{a(\mathbf{r})=0}^{N} a^{2}(\mathbf{r}) X(a(\mathbf{r}), n),
\end{aligned}
$$

where $\left(\hat{W}^{D}\right)^{p}$ is to be understood as a time-ordered product of operators $\hat{W}^{D}$ taken at any $p$ moments of time between 0 and $n . X(a(\mathbf{r}), n)$ is any function of $a(\mathbf{r})$ and time which has the following properties:

$$
\sum_{a(\mathbf{r})=0}^{N} X(a(\mathbf{r}), n)=0,
$$

$$
\sum_{a(\mathbf{r})=0}^{N} a(\mathbf{r}) X(a(\mathbf{r}), n)=0 .
$$

We remove one of the operators $\hat{W}^{D}$ by adding the inverse operator $\left(\hat{W}^{D}\right)^{-1}$, taken at an appropriate moment of time, to the left of $\left(\hat{W}^{D}\right)^{p}$ in (25). Bearing in mind the property (25), we find

$$
\begin{aligned}
& \sum_{a(\mathbf{r})=0}^{N} a(\mathbf{r}) \hat{W}^{D^{-1}}\left(\hat{W}^{D}\right)^{p} X(a(\mathbf{r}), n)=\left(-\frac{1}{N}\right)^{p-1} \sum_{a(\mathbf{r})=0}^{N} a(\mathbf{r}) X(a(\mathbf{r}), n), \\
& \sum_{a(\mathbf{r})=0}^{N} a^{2}(\mathbf{r}) \hat{W}^{D^{-1}}\left(\hat{W}^{D}\right)^{p} X(a(\mathbf{r}), n)=\left(-\frac{2}{N}\right)^{p-1} \sum_{a(\mathbf{r})=0}^{N} a^{2}(\mathbf{r}) X(a(\mathbf{r}), n) .
\end{aligned}
$$


Note that if $(26)$ holds for $X(a(\mathbf{r}), n)$, it also holds for $\left(\hat{W}^{D}\right)^{p} X(a(\mathbf{r}), n)$, i.e. $\quad \hat{W}^{D}$ is a function-to-function map within the class of functions satisfying (26). Furthermore, $\hat{S} P_{B}(a(\mathbf{r}), n)$ belongs to that class of functions, as can be easily seen from the definition of the operator $\hat{S}(13)$ and the following two identities:

$$
\begin{aligned}
& \sum_{a(\mathbf{r})=0}^{N} a(\mathbf{r}) \hat{W}^{R} P_{B}(a(\mathbf{r}), n)=R(\bar{a}(\mathbf{r}, n)), \\
& \sum_{a(\mathbf{r})=0}^{N} a(\mathbf{r}) P_{B}(a(\mathbf{r}), n)=\bar{a}(\mathbf{r}, n) .
\end{aligned}
$$

Using these observations, we can infer by simple inspection of (25) and (27) that

$$
\begin{aligned}
\sum_{a(\mathbf{r})=0}^{N} a(\mathbf{r})\left(\hat{W}^{D}\right)^{-1} \hat{S} P_{B}(a(\mathbf{r}), n) & =-N \sum_{a(\mathbf{r})=0}^{N} a(\mathbf{r}) \hat{S} P_{B}(a(\mathbf{r}), n) \\
& =0 \\
\sum_{a(\mathbf{r})=0}^{N} a^{2}(\mathbf{r})\left(\hat{W}^{D}\right)^{-1} \hat{S} P_{B}(a(\mathbf{r}), n) & =-\frac{N}{2} \sum_{a(\mathbf{r})=0}^{N} a^{2}(\mathbf{r}) \hat{S} P_{B}(a(\mathbf{r}), n) .
\end{aligned}
$$

Using (7) and (29), after a few simple transformations, we obtain

$$
\begin{aligned}
\sum_{a(\mathbf{r})=0}^{N} a(\mathbf{r}) \hat{W}^{R}\left(\hat{W}^{D}\right)^{-1} \hat{S} P_{B}(a(\mathbf{r}), n) & =h \sum_{a(\mathbf{r})=0}^{N}\left[\left(\frac{N}{N-1}-k_{2}\right) a(\mathbf{r})-\frac{h}{N-1} a^{2}(\mathbf{r})\right]\left(\hat{W}^{D}\right)^{-1} \hat{S} P_{B}(a(\mathbf{r}), n) \\
& =\frac{N h}{2(N-1)} \sum_{a(\mathbf{r})=0}^{N} a^{2}(\mathbf{r}) \hat{S} P_{B}(a(\mathbf{r}), n) \\
& =\frac{N h^{2}}{N-1} \bar{a}(\mathbf{r}, n)\left(1-\frac{\bar{a}(\mathbf{r}, n)}{N}\right)^{2}
\end{aligned}
$$

for the $\mathcal{O}\left(\gamma^{2}\right)$ correction term.

[1] P. Fife, Mathematical Aspects of Reacting and Diffusing Systems, Lecture Notes in Biomathematics, Vol. 28 (Springer, New York, 1979).

[2] Oscillations and Travelling Waves in Chemical Systems, R.J. Field and M. Burger, eds., (Wiley, New York, 1985).

[3] Nonequilibrium Chemical Dynamics: From Experiment to Microscopic Simulations, F. Baras and D. Walgraef, eds., Physica A 188 (1992).

[4] A.S. Mikhailov, Foundations of Synergetics I. Distributed Active Systems, (Springer-Verlag, Berlin, 1994).

[5] R.A. Fisher, Ann. Eugenics, 7, 335 (1937).

[6] A. Kolmogorov, I. Petrovsky, and N. Piskunov, Bull. Univ. Moscow Ser. Int. Sec. A 1, 1 (1937).

[7] S. Cornell, M. Droz, B. Chopard, Phys. Rev. A, 44, 4826 (1991); B. Chopard and M. Droz, Europhys. Lett., 15, 459 (1991).

[8] S.K. Scott and K. Showalter, in Chemical Waves and
Patterns, R. Kapral and K. Showalter, eds. (Kluwer Academic, Dordrecht, 1995).

[9] J.D. Murray, Mathematical Biology, (Springer-Verlag, Berlin, 1989).

[10] Ya. B. Zeldovich and G.I. Barenblatt, Combust. Flame, 3, 61 (1959).

[11] J. Billingham and D.J. Needham, Phil. Trans. R. Soc. Lond. A, 334, 1 (1991).

[12] A. Lemarchand, H. Lemarchand, A. Lesne, and M. Mareschal, in Far From Equilibrium Dynamics of Chemical Systems III, J. Gorecki et al., eds. (World Scientific, Singapore, 1994).

[13] A. Lemarchand, A. Lesne, M. Mareschal, Phys. Rev. E, 51, 4457 (1995).

[14] M.A. Karzazi, A. Lemarchand, M. Mareschal, Phys. Rev. E, 54, 4888 (1996).

[15] J. Gorecki and J.N. Gorecka, Acta Physica Pol. B, 29, 1663 (1998).

[16] R.H. Goodman, D.S. Graff, L.M. Sanders, P. LerouxHugon, E. Clément, Phys. Rev. E, 52, 5904 (1995).

[17] E. Brunet, B. Derrida, Phys. Rev. E, 56, 2597 (1997).

[18] M.V. Velikanov and R. Kapral, J. Chem. Phys., 109, 281 (1998). 
[19] D.R. Cox and H.D. Miller, The Theory of Stochastic Processes, (Methuen, London, 1965).

[20] C.W. Gardiner, Handbook of Stochastic Methods for Physics, Chemistry and the Natural Sciences, (SpringerVerlag, Berlin, 1985).

[21] G. Uhlenbeck, Lectures in Statistical Mechanics, (Am. Math. Soc., Providence, RI, 1963); S. Chapman and
T. G. Cowling, Mathematical Theory of Non-Uniform Gases, (Cambridge Univ. Press, London, 1961).

[22] A. Malevanets and R. Kapral, Phys. Rev. E., 55, 5657 (1997)

[23] J. Riordan, C.R. Doering, D. ben-Avraham, Phys. Rev. Lett., 75, 565 (1995). 